\title{
Prophylaxis for deep venous thrombosis in neurosurgical oncology: review of 2779 admissions over a 9-year period
}

\author{
Sarah F. Smith, B.Sc., M.P.H.(Hons), Judy M. Simpson, Ph.D., C.Stat., \\ and Lali H.S. Sekhon, M.B.,B.S.,(Hons), F.R.A.C.S., PH.D.
}

Department of Neurosurgery, Royal North Shore Hospital, St. Leonards; and School of Public Health, University of Sydney, New South Wales, Australia

\begin{abstract}
Object. Management of disease in patients undergoing neurosurgical treatment for tumors requires balancing the competing risks of hemorrhage and thrombosis. The authors compared the incidence of clinically apparent deep venous thrombosis (DVT) and pulmonary embolism (PE) in patients admitted for treatment of intracranial and spinal tumors at three institutions. At the public hospital (the Royal North Shore Hospital [RNSH]) nonsequential calf compression was used, and at the other two private neurosurgery services sequential calf compression with low-molecular-weight heparin was used in patients undergoing spinal surgery. All patients wore compression stockings and underwent follow-up scanning after surgery.

Methods. The authors identified from their neurosurgery databases 2779 discharges of patients with tumor from the RNSH and private hospitals between January 1, 1995 and December 31, 2003. Patient admissions were relatively well matched for age, sex, duration of stay, and tumor type. For patients who underwent spinal surgery, the incidence of DVT was higher in the RNSH (2.6\% of admissions) than in private hospitals, where no case of DVT was seen ( $p=$ 0.02). The incidence of PE was higher in admissions patients who had been treated for cranial tumors in the RNSH $(2.9 \%)$ than in those treated in the private hospitals $(1.3 \%, \mathrm{p}=0.01)$.

Possible reasons for these discrepancies include a higher proportion of ambulatory patients before and after surgery in the group treated at private hospitals. More emergency and semi-emergency surgery was performed in the RNSH than in the private hospitals. Nevertheless, fewer patients discharged from the RNSH had undergone surgery, which is a known risk factor for DVT.

Conclusions. Patients with intracranial tumors had a higher incidence of PE than those with spinal tumors. The incidence of DVT and PE was higher in patients admitted to the public hospital. The incidence of DVT in patients undergoing spinal procedures was lower when low-molecular-weight heparin was used judiciously, but the incidence of PE in patients undergoing cranial procedures was lower with the private hospital protocol, which did not include prophylaxis with anticoagulating agents.
\end{abstract}

\section{KEY WORDS • brain tumor • deep venous thrombosis • neurosurgery • prophylaxis • pulmonary embolism • spinal tumor}

\begin{abstract}
All neurosurgeons fear hemorrhage in their patients after cranial or spinal surgery, but this is balanced by the contrasting fear of DVT and PE. Prophylaxis for DVT and PE is problematic for neurosurgeons. Despite multiple studies of prevention conducted over the last 30 years as well as the development of low-molecular-weight heparins and mechanical prophylaxis (sequential calf compression devices, calf stimulators, and antithromboembolism stockings), DVT is the most frequent systemic complication in patients undergoing neurosurgery, ${ }^{11}$ and PE can still be fatal. ${ }^{6}$ Although several studies have shown that minidose heparin (5000 U administered subcutaneously twice daily) or low-molecular-weight heparin can be used for DVT prophylaxis without increasing the risk of hemorrhage in patients who undergo neurosurgery, ${ }^{1,7}$ some
\end{abstract}

Abbreviations used in this paper: DVT = deep venous thrombosis; $\mathrm{PE}=$ pulmonary embolism; RNSH = Royal North Shore Hospital. neurosurgeons are wary of using anticoagulant drugs for prevention of venous thromboembolism. ${ }^{2,5}$

In a previous study conducted at one of our institutions, a significant increase was found in the incidence of DVT between the late 1970s and the late 1990s, and intracranial tumors were the diagnostic group with the second-highest incidence of DVT and PE. ${ }^{9}$ Because neurosurgical procedures and malignant tumors are known risk factors for development of venous thromboembolism, we compared the incidence of DVT and PE in patients treated for intracranial and spinal tumors at one public and two private specialist neurosurgery services where different DVT prophylaxis methods were used over the last 9 years.

\section{CLINICAL MATERIAL AND METHODS}

\section{Patient Population}

The neurosurgical database containing information on 
S. F. Smith, J. M. Simpson, and L. H. S. Sekhon

more than 25,000 admissions over a 25 -year period at the RNSH, a public teaching hospital, has been described previously. ${ }^{10}$ From this group, and from similar comprehensive databases maintained in nearby Dalcross Private Hospital and North Shore Private Hospital, a total of 2779 patients were identified who were discharged between January 1, 1995, and December 31, 2003, after treatment for extradural or intradural spinal or intracranial tumors. There were no exclusions. Significantly, the same surgeons who admitted patients to the public hospital also cared for the patients in the private hospitals, and all surgery was performed by one of five neurosurgeons.

\section{Treatment Protocols}

Public Hospital. Patients undergoing surgery at the RNSH received nonsequential calf compression during surgery and until discharge from the intensive care unit. Follow-up Doppler ultrasonography studies were performed once weekly in the intensive care unit and on the general neurosurgery ward within 5 to 10 days postoperatively. All patients wore below-knee surgical antithromboembolism stockings until they were discharged.

Private Hospitals. Patients undergoing surgery at either private hospital received mechanical sequential thighlength compression during surgery and during their intensive care unit stay, and these devices remained in situ until the patients were mobilized. Ninety percent of patients undergoing spinal surgery received the low-molecular-weight heparin dalteparin sodium (2500 U daily) beginning with the first postoperative day and continuing for 14 days or until discharge, whichever came first. Patients were encouraged to move their toes daily until they were discharged. All patients wore below-knee surgical antithromboembolism stockings during their entire hospital stay.

At all institutions Doppler follow-up ultrasonography studies were performed routinely within the 1 st week postoperatively. The diagnosis of DVT was made in all hospitals based on clinical findings of leg and/or arm swelling or pain and was confirmed with venous Doppler ultrasonography. The diagnosis of PE was made based on clinical findings of chest pain and/or breathlessness, and was confirmed by evidence of a medium to high probability of embolism on V/Q scanning or spiral computerized tomography pulmonary angiography.

\section{RESULTS}

Admissions for tumor to the RNSH and private hospitals were approximately equal in number, with $14 \%$ after treatment for extra- or intradural spinal tumors. Patients with tumors at the RNSH were slightly younger (by 1.7 years) than patients with tumors at the private hospitals, a clinically insignificant difference, although it was statistically significant because of the large numbers. Similarly, patients with tumors at the RNSH had a longer stay (by 1.4 days) than those at the private hospitals, which is unlikely to be clinically significant, and the proportion of female patients did not differ in the cranial, spinal, or combined tumor groups (Table 1). In $23.6 \%$ of RNSH and $9.5 \%$ of private hospital admissions for tumors, surgery
TABLE 1

Characteristics of admissions of patients with tumor to public and private hospitals*

\begin{tabular}{lccl}
\hline \hline \multicolumn{1}{c}{ Factor } & $\begin{array}{c}\text { Public } \\
\text { Hospital }\end{array}$ & $\begin{array}{c}\text { Private } \\
\text { Hospitals }\end{array}$ & $\mathrm{z} \chi^{2}$ for Difference, $\mathrm{p}$ Value \\
\hline all patients & 1451 & $1328 \dagger$ & \\
mean age (yrs) & 55.4 & 57.1 & $\mathrm{z}=-2.83, \mathrm{p}=0.005$ \\
\% female & 48.2 & 49.3 & $\chi^{2}=0.28,1 \mathrm{df}, \mathrm{p}=0.6$ \\
geo mean LOS (days) & 10.3 & 8.9 & $\mathrm{z}=4.98, \mathrm{p}<0.0001$ \\
\% w/o op & 23.6 & 9.5 & $\chi^{2}=98.2,1 \mathrm{df}, \mathrm{p}<0.0001$ \\
all w/cranial tumors & 1259 & $1129 \dagger$ & \\
mean age (yrs) & 55.3 & 57.3 & $\mathrm{z}=-3.12, \mathrm{p}=0.002$ \\
\% female & 48.6 & 49.3 & $\chi^{2}=0.13,1 \mathrm{df}, \mathrm{p}=0.7$ \\
geo mean LOS (days) & 10.1 & 8.9 & $\mathrm{z}=4.42, \mathrm{p}<0.0001$ \\
\% w/o op & 22.4 & 10.2 & $\chi^{2}=64.1,1 \mathrm{df}, \mathrm{p}<0.0001$ \\
all w/spinal tumors & 192 & $199 \dagger$ & \\
mean age (yrs) & 57.9 & 54.8 & $\mathrm{z}=1.23, \mathrm{p}=0.2$ \\
$\%$ female & 45.3 & 48.2 & $\chi^{2}=0.34,1 \mathrm{df}, \mathrm{p}=0.6$ \\
geo mean LOS (days) & 11.1 & 9.1 & $\mathrm{z}=2.28, \mathrm{p}=0.02$ \\
\% w/o op & 28.9 & 6.4 & $\chi^{2}=5.9,1 \mathrm{df}, \mathrm{p}=0.02$ \\
\hline
\end{tabular}

$*$ Df = degrees of freedom; geo = geometric; LOS = length of stay.

$\dagger$ Ages were not available for a few discharged patients and were omitted.

was not performed. Patients in $1.9 \%$ of all tumor admissions from the RNSH and $1.3 \%$ of all tumor discharges from the private hospitals experienced post- or perioperative hemorrhage.

Whereas the rate of DVT in discharged patients with tumors in either location and in the subgroup with cranial tumors was similar for those treated at public and private hospitals, the DVT rate was significantly higher for patients with spinal tumors who were treated at the RNSH (Table 2). In contrast, no DVT was diagnosed in 229 admissions after surgery for spinal tumors at the private hospitals. The rate of PE was significantly higher in patients with cranial tumors treated in the public hospital, but not for those with spinal tumors, for which the numbers were very small. At $5 \%$ of admissions in both hospital groups, meningioma was the tumor associated with the highest incidence of clinical DVT. High-grade glial tumors were associated with twice the rate of DVT and of $\mathrm{PE}$ in the RNSH compared with the private institutions.

\section{DISCUSSION}

We found a $2.7 \%$ rate of DVT in patient admissions for all hospitals combined. In a previous study ${ }^{9}$ of patients treated at the RNSH between 1979 and 1994, before the splitting of neurosurgical admissions between public and private hospitals, the rate of DVT in patients with neurosurgically treated tumors rose from $0.7 \%$ of admissions between 1979 and 1983 to $1.8 \%$ of admissions between 1989 and 1993. This increase may be the result of more frequent screening with Doppler ultrasonography since the mid-1990s. Increasing age in the patient population and the trend toward diagnosis and treatment of older patients with tumors ${ }^{10}$ would also influence the rate of DVT, because increasing age has been shown to be highly predictive of this disorder.

For cranial tumors, our findings of DVT incidences of $3.2 \%$ (public hospital) and $2.8 \%$ (private hospitals) are lower than those of Dickinson, et al., although these au- 
Prophylaxis for DVT in neurosurgical oncology: 9-year review

TABLE 2

Frequency of DVT and PE for major tumor types in 2779 admissions to public and private hospitals*

\begin{tabular}{|c|c|c|c|c|c|c|c|}
\hline \multirow[b]{2}{*}{ Tumor Type } & \multicolumn{3}{|c|}{ Public Hospital } & \multicolumn{3}{|c|}{ Private Hospitals } & \multirow[b]{2}{*}{$\mathrm{p}$ Value $\dagger$} \\
\hline & $\begin{array}{c}\text { Discharges } \\
(\%)\end{array}$ & $\begin{array}{c}\text { No. w/ } \\
\text { DVT (\%) }\end{array}$ & $\begin{array}{l}\text { No. w/ } \\
\text { PE (\%) }\end{array}$ & $\begin{array}{c}\text { Discharges } \\
(\%)\end{array}$ & $\begin{array}{c}\text { No. w/ } \\
\text { DVT (\%) }\end{array}$ & $\begin{array}{l}\text { No. w/ } \\
\text { PE (\%) }\end{array}$ & \\
\hline all & 1452 & $45(3.1)$ & $38(2.6)$ & 1379 & $32(2.3)$ & $16(1.2)$ & $0.2,0.006$ \\
\hline all cranial & 1259 & $40(3.2)$ & $36(2.9)$ & 1150 & $32(2.8)$ & $15(1.3)$ & $0.6,0.01$ \\
\hline high-grade glial & $358(28.5)$ & $16(4.5)$ & $17(4.8)$ & 338 (29.4) & $8(2.5)$ & $7(2.2)$ & \\
\hline meningioma & $241(19.1)$ & $13(5.4)$ & $5(2.1)$ & $240(20.9)$ & $13(5.4)$ & $2(0.8)$ & \\
\hline metastasis & $213(16.9)$ & $4(1.9)$ & $8(3.8)$ & $200(17.4)$ & $7(3.5)$ & $6(3.0)$ & \\
\hline other cranial $\ddagger$ & 447 (35.5) & $7(1.6)$ & $6(1.3)$ & $372(32.3)$ & $3(0.9)$ & $0(0.0)$ & \\
\hline all spinal & 194 & $5(2.6)$ & $2(1.0)$ & 229 & 0 & $1(0.4)$ & $0.02,0.6$ \\
\hline extradural & $129(66.5)$ & $2(1.6)$ & $2(1.6)$ & $125(54.6)$ & 0 & 0 & \\
\hline intradural & $65(33.5)$ & $3(4.6)$ & $0(0.0)$ & $104(45.4)$ & 0 & $1(1.0)$ & \\
\hline
\end{tabular}

* Discharge totals for Table 2 do not equal those for Table 1 because some patients had multiple tumor diagnoses.

$\dagger$ Probability value for difference between public and private hospitals for DVT and PE, respectively, according to the Fisher exact test.

‡ Includes acoustic neuroma, craniopharyngioma, pituitary tumor, ungradable glioma, pineal tumor, ependymoma, medulloblastoma, neurofibroma, hemangioma, and colloid cyst.

thors excluded pituitary and skull base tumors. Sawaya, et al., ${ }^{8}$ report a low clinically confirmed DVT rate of $1.25 \%$ and a PE rate of $0.25 \%$ after intraaxial cranial tumor resections. Few reports exist in the literature regarding the incidence of DVT and PE after spinal tumor surgery. Gerlach, et al., ${ }^{4}$ report only one case of DVT and none of PE in a series of 1954 patients who underwent spinal operations for neurosurgical indications.

The possible explanations for the higher rate of DVT/ $\mathrm{PE}$ in patients treated in the public hospital are severalfold. In the public hospital, surgery is often performed on an emergency/semi-emergency basis. Prophylaxis was less rigorous and consistent in the patients treated in the public hospital. These individuals were often less ambulatory both before and after surgery, and the incidence of paraplegia/ quadriplegia before and after surgery was higher in patients treated in the public hospital. Nevertheless, fewer patients admitted to the public hospital underwent surgery, which is a known risk factor for DVT.

\section{CONCLUSIONS}

Low rates of clinically apparent DVT and PE can be achieved without the use of low-molecular-weight heparin in all patients admitted for neurosurgical care. Our comparison of protocols shows that the incidence of DVT was higher in patients treated in the public hospital, where prophylaxis did not include the use of intraoperative sequential calf compression or low-molecular-weight heparin. Patients with intracranial tumors had a higher incidence of PE than those with spinal tumors. For patients undergoing cranial surgery, a lower incidence of PE was achieved in the private hospitals with sequential calf compression and without the use of prophylactic anticoagulant drugs. For those undergoing spinal surgery, a lower incidence of DVT was achieved in the private hospitals with careful postoperative use of low-molecular-weight heparins.

\section{References}

1. Agnelli G, Piovella F, Buoncristiani P, et al: Enoxaparin plus compression stockings compared with compression stockings alone in the prevention of venous thromboembolism after elective neurosurgery. N Engl J Med 339:80-85, 1998

2. Carman TL, Kanner AA, Barnett GH, et al: Prevention of thromboembolism after neurosurgery for brain and spinal tumors. South Med J 96:17-22, 2003

3. Dickinson LD, Miller LD, Patel CP, et al: Enoxaparin increases the incidence of postoperative intracranial hemorrhage when initiated preoperatively for deep venous thrombosis prophylaxis in patients with brain tumors. Neurosurgery 43:1074-1081, 1998

4. Gerlach R, Raabe A, Beck J, et al: Postoperative nadroparin administration for prophylaxis of thromboembolic events is not associated with an increased risk of hemorrhage after spinal surgery. Eur Spine J 13:9-13, 2004

5. Gnanalingham KK, Holland JP: Attitudes to the use of prophylaxis for thrombo-embolism in neurosurgical patients. J Clin Neurosci 10:467-469, 2003

6. Hamilton MG, Hull RD, Pineo GF: Venous thromboembolism in neurosurgery and neurology patients: a review. Neurosurgery 34:280-296, 1994

7. Macdonald RL, Amidei C, Lin G, et al: Safety of perioperative subcutaneous heparin for prophylaxis of venous thromboembolism in patients undergoing craniotomy. Neurosurgery 45: 245-252, 1999

8. Sawaya R, Hammoud M, Schoppa D, et al: Neurosurgical outcomes in a modern series of 400 craniotomies for treatment of parenchymal tumors. Neurosurgery 42:1044-1056, 1998

9. Smith SF: Influences on the Incidence of Clinical Deep Vein Thrombosis and Pulmonary Embolism in a Prospectively Collated Population of 21000 Neurosurgical Inpatients. Thesis. Sydney: University of Sydney, 2001

10. Smith SF, Simpson JM, Sekhon LH: A quarter of a century of neurosurgery: the value of a relational database to document trends in neurosurgical practice of a tertiary referral hospital. J Clin Neurosci 11:31-36, 2004

11. Warnick RE: Surgical complications and their avoidance, in Winn HR (ed): Youmans Neurological Surgery, ed 5. Philadelphia: Saunders, 2004, pp 931-940

Manuscript received August 23, 2004.

Accepted in final form September 7, 2004.

Ms. Smith receives funding from the Andrew Olle Memorial Trust.

Address reprint requests to: Sarah F. Smith, Department of Neurosurgery, Royal North Shore Hospital,St. Leonards, New South Wales 2065, Australia. email: sfsmith@doh.health.nsw.gov.au. 